OPEN ACCESS

Edited by:

Ayrton R. Massaro,

Hospital Sírio-Libanês, Brazil

Reviewed by:

Mirjam R. Heldner,

University Hospital Bern, Switzerland

Silvia Schönenberger,

Heidelberg University Hospital,

Germany

*Correspondence:

Min Lou

Im99@zju.edu.cn;

loumingxc@vip.sina.com

tThese authors have contributed equally to this work

Specialty section: This article was submitted to

Stroke,

a section of the journa

Frontiers in Neurology

Received: 27 October 2018 Accepted: 29 March 2019

Published: 17 April 2019

Citation:

Gong X, Chen Z, Shi F, Zhang M, Xu C, Zhang R and Lou M (2019)

Conveniently-Grasped Field Assessment Stroke Triage (CG-FAST): A Modified Scale to Detect Large Vessel Occlusion Stroke.

Front. Neurol. 10:390.

doi: 10.3389/fneur.2019.00390

\section{Conveniently-Grasped Field Assessment Stroke Triage (CG-FAST): A Modified Scale to Detect Large Vessel Occlusion Stroke}

\author{
Xiaoxian Gong ${ }^{1 \dagger}$, Zhicai Chen ${ }^{1 \dagger}$, Feina Shi ${ }^{1}$, Meixia Zhang ${ }^{2}$, Chao Xu ${ }^{1}$, Ruiting Zhang ${ }^{1}$ and \\ Min Lou ${ }^{1,3 *}$

\footnotetext{
'Department of Neurology, The Second Affiliated Hospital of Zhejiang University, School of Medicine, Hangzhou, China, ${ }^{2}$ Department of Neurology, Jinhua Hospital of Zhejiang University, Jinhua, China, ${ }^{3}$ Zhejiang University Brain Research Institute, Hangzhou, China
}

Background and Purpose: Patients with large vessel occlusion stroke (LVOS) need to be rapidly identified and transferred to comprehensive stroke centers (CSC). However, previous pre-hospital strategy remains challenging. We aimed to develop a modified scale to better predict LVOS.

Methods: We retrospectively reviewed our prospectively collected database for acute ischemic stroke (AIS) patients who underwent CT angiography (CTA) or time of flight MR angiography (TOF-MRA) and had a detailed National Institutes of Health Stroke Scale (NIHSS) score at admission. Large vessel occlusion (LVO) was defined as the complete occlusion of large vessels, including the intracranial internal carotid artery (ICA), M1, and M2 segments of the middle cerebral artery (MCA), and basilar artery (BA). The Conveniently-Grasped Field Assessment Stroke Triage (CG-FAST) scale consisted of Level of Consciousness (LOC) questions, Gaze deviation, Facial palsy, Arm weakness, and Speech changes. Receiver Operating Characteristic (ROC) analysis was used to obtain the Area Under the Curve (AUC) of CG-FAST and previously established pre-hospital prediction scales.

Results: Finally, 1,355 patients were included in the analysis. LVOS was detected in 664 (49.0\%) patients. The sensitivity, specificity, positive predictive value, and negative predictive value of CG-FAST were $0.617,0.810,0.785$, and 0.692 respectively, at the optimal cutoff $(\geq 4)$. The AUC, Youden index and accuracy of the CG-FAST scale $(0.758$, 0.428 , and 0.728) were all higher than other pre-hospital prediction scales.

Conclusions: CG-FAST scale could be an effective and simple scale for accurate identification of LVOS among AIS patients.

Keywords: large artery occlusion, stroke, endovascular treatment, NIHSS, scale 


\section{INTRODUCTION}

Large vessel occlusion stroke (LVOS) often leads to severe disability and mortality. Although endovascular therapy (EVT) has been proved to be effective for LVOS patients (19 ), especially in anterior circulation, its benefit is highly time-dependent $(10,11)$. As hospitals with around-the-clock endovascular capability are scarce in many parts of the world and patients admitted directly to a CSC would have better outcomes than those receiving drip and ship treatment $(12,13)$, this requirement presents a big challenge to current systems of delivering appropriate patients to comprehensive stroke centers (CSC). It is thus critical for emergency paramedics to precisely identify LVOS in the setting of pre-hospital triage stage $(14,15)$.

Current guideline recommends the use of National Institutes of Health Stroke Scale (NIHSS) scores $\geq 6$ to select patients for EVT (16), as patients with acute LVOS are strongly associated with high NIHSS (17-19). However, in the prehospital triage stage, it is not very practical to complete the whole examination of NIHSS. Actually, certain items of NIHSS may be more informative of LVOS than a simple score, which usually reflects the overall severity of deficits. Thus, several examination tools derived from NIHSS have been proposed for use in the pre-hospital setting (14, 20-25), for example, recent G-FAST score (Gaze, Face, Arm, and Speech) has been shown to improve the predictive capability of LVOS when compared to other tools (25). Based on our clinical observation, we found that the level of consciousness, which was not included in G-FAST, was easier to be disturbed in LVOS patients and it had relatively high specificity to indicate the presence of LVOS when combined with other neurological deficits. We posit the addition of the item "consciousness level" to the G-FAST score may increase the predictive ability. Thus, we developed a new simple scale derived from the GFAST score, named the Conveniently-Grasped Field Assessment Stroke Triage (CG-FAST) scale and then designed a test to determine whether the CG-FAST scale could achieve a better predictive performance than the preexisting scales for accurate identification of LVOS.

\section{MATERIALS AND METHODS}

\section{Subjects and Methods}

We retrospectively reviewed our prospectively collected database for acute stroke patients (hemorrhagic or ischemic stroke) within $8 \mathrm{~h}$ of onset in our center during period from June 2009 to July 2018. We enrolled patients who (1) underwent CT angiography (CTA) or time of flight MR angiography (TOF-MRA) within $8 \mathrm{~h}$, (2) had a detailed NIHSS at admission, (3) had a diagnosis of AIS confirmed by diffusion-weighted imaging or CT at $24 \mathrm{~h}$ after symptom onset. Patients with poor image quality due to motion artifacts were excluded. Demographic, clinical, and laboratory data were recorded including age, gender; prior antiplatelet therapy; risk factors (smoking, hypertension, diabetes mellitus,
TABLE 1 | The CG-FAST scale and its correspondence to the NIHSS

\begin{tabular}{lcc}
\hline Item & CG-FAST score & NIHSS score source \\
\hline LOC QUESTIONS & 0 & 0 \\
Normal & 1 & $1-2$ \\
One correct or neither correct & 0 & 0 \\
GAZE & 1 & $1-2$ \\
Normal & & \\
Partial or forced deviation & 0 & 0 \\
FACIAL PALSY & 1 & $1-3$ \\
Normal & & $0-1$ \\
Minor, partial, or complete paralysis & 0 & $2-4$ \\
ARM WEAKNESS & 1 & \\
No drift or drift but doesn't hit bed & & 0 \\
Some effort against gravity, no effort & & $1-3 / 1-2$ \\
against gravity or no movement & 0 & \\
SPEECH PROBLEMS & 1 & \\
Normal & & \\
Aphasia or dysarthria & &
\end{tabular}

CG-FAST indicates Conveniently-Grasped Field Assessment Stroke Triage; LOC, level of consciousness; and NIHSS, National Institutes of Health Stroke Scale.

hyperlipidemia, history of stroke/TIA, and atrial fibrillation); blood pressure and blood glucose.

As shown in Table 1, the CG-FAST scale (LOC Questions [scored 0-1], Gaze [0-1], Facial palsy [scored 0-1], Arm weakness $[0-1]$, and Speech problems [0-1]) was graded as detailed and derived from the NIHSS assessed by experienced neurologists at hospital admission.

LVOS in current study was defined as unilateral occlusion of intracranial internal carotid artery (ICA), M1, and M2 segments of the middle cerebral artery (MCA), and basilar artery (BA) $(14,26)$ on baseline CTA or TOFMRA. Two experienced neurologists blinded to the patients' information assessed the occlusion on CTA or TOF-MRA at admission with rater discrepancies settled by consensus.

\section{Statistical Methods}

Patients were dichotomized into a LVOS group and a nonLVOS group. Clinical characteristics were summarized by computing the mean \pm standard deviation (SD) or median (interquartile range), and differences between the two groups were estimated by the $t$-test or Mann-Whitney $U$-test if they were continuous variables. Categorical or binary datum was summarized by proportion (n); and differences between the two groups were estimated by the Pearson $\chi^{2}$ test. Receiver operating characteristic (ROC) analysis was used to get the area under the curve (AUC) of each pre-hospital prediction scale. The optimal cutoff was determined at the maximal Youden Index (27). Sensitivity, specificity, positive predictive value (PPV), negative predictive value (NPV), and accuracy were calculated for the prediction of LVOS. All statistical analysis was performed using SPSS, Version 22.0 (IBM, Armonk, New York). A $P<0.05$ was considered statistically significant. 


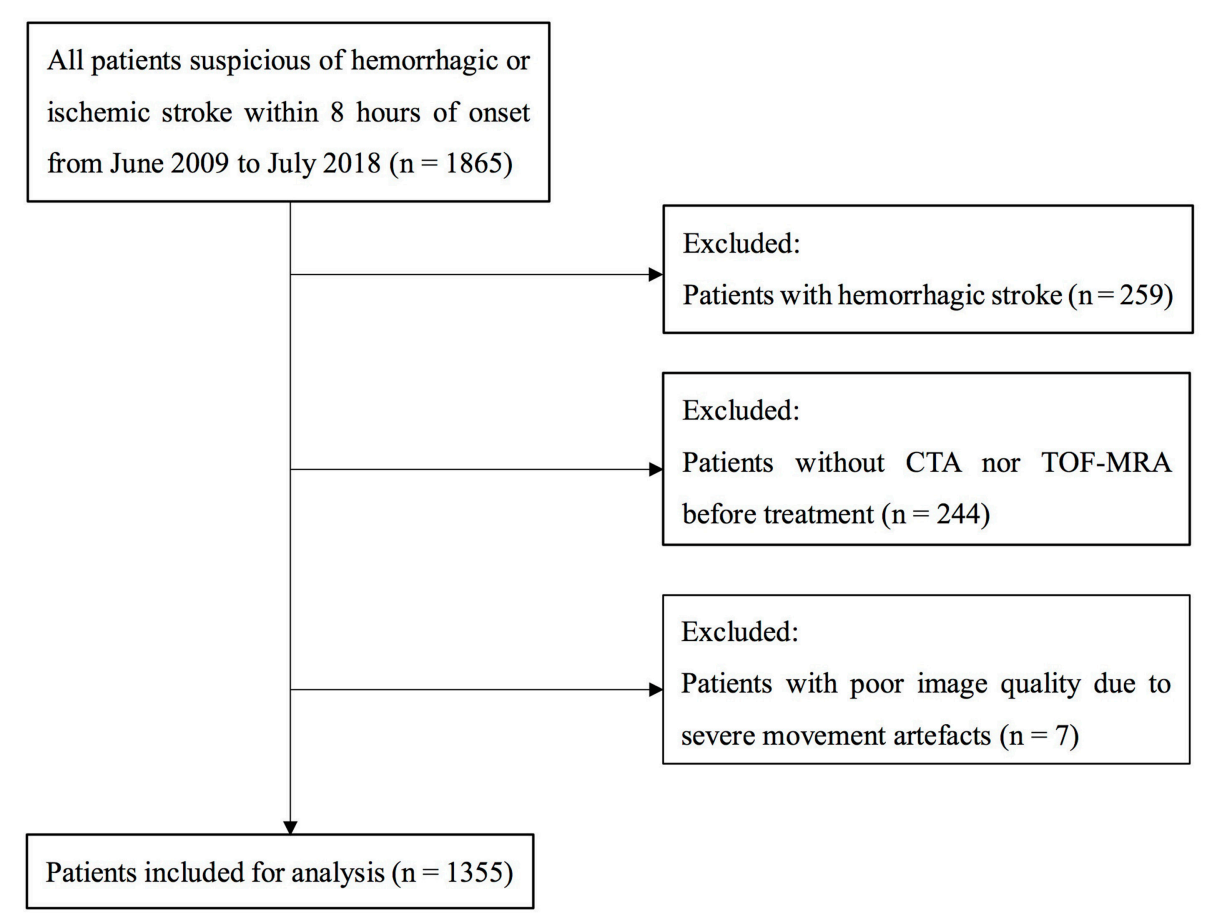

FIGURE 1 | Flow chart of patient selection. CTA indicates CT angiography; and TOF-MRA, time of flight MR angiography.

\section{RESULTS}

A total of 1355 patients were finally included in the analysis. The flow chart of patient selection is illustrated in Figure 1. Of the included patients, mean age was $69 \pm 13$ years and $821(60.6 \%)$ were male. Median NIHSS on admission was 8 (3-15). Among them, $664(49.0 \%)$ patients had LVOS and 691 (51.0\%) were in the non-LVOS group.

Table 2 shows that patients with LVOS were older $(p=0.001)$, had higher rate of atrial fibrillation $(p<0.001)$, lower rate of diabetes mellitus $(p=0.018)$, lower systolic blood pressure ( $p$ $<0.001)$, lower blood glucose $(p=0.025)$, and higher NIHSS score $(p<0.001)$, compared with non-LVOS patients. Besides, the scores of LOC questions, gaze, facial palsy, arm weakness, and speech changes were significantly higher in the LVOS group.

The AUC of CG-FAST scale was 0.758. Figure 2 shows different cutoff values of the CG-FAST for predicting LVOS. Highest Youden Index was achieved for score $\geq 4$, with sensitivity, specificity, PPV and NPV of $0.617,0.810,0.785$, and 0.692 , respectively. The diagnostic parameters of CG-FAST were compared with preexisting scales including FAST-ED, 3ISS, CPSSS, PASS, RACE, LAMS, G-FAST, and NIHSS (Table 3). The AUC and highest Youden index of the CG-FAST scale were higher than other preexisting scales.

\section{DISCUSSION}

The current study indicated that our novel CG-FAST scale could be effective for accurate identification of LVOS among AIS patients, as its predictive value for LVOS was even higher than other preexisting scales, as well as NIHSS.

There are several advantages of the CG-FAST scale over preexisting scales, which may explain its relatively high predictive value for LVOS. The evaluation of LOC is relatively complicated for emergency paramedics, especially when patients present with somnolence, sopor state or coma. We thus adopted LOC questions in the CG-FAST, which is easier and could be combined with the evaluation of aphasia and dysarthria. Actually, most of the previous pre-hospital scales ignored the evaluation of aphasia or dysarthria (14, 20-24), which would miss some typical signs of cortical, subcortical, or brain stem dysfunction, the possible discriminators of LVOS. Besides, different from the previous 3-ISS, RACE and LAMS scales, which gave a higher weight to motor symptoms, CG-FAST only preserved the simple arm weakness score based upon the consideration that motor symptoms can also occur in lacunar stroke and thus are not good indicators for LVOS, though they strongly relate to the severity of stroke. And neglect was not contained in CG-FAST due to its low inter-rater reliability of evaluation in emergency conditions, though it was reported to be associated with large cortical infarct (14). Instead, we recruited gaze deviation as it could be easily observed by paramedics and was regarded as a typical sign of large artery occlusion in the anterior or posterior circulation (28-30). Last but not least, the method of dichotomy for CG-FAST was simple as no extra ranking information was needed except for the confirmation of presence or absence of symptoms. Moreover, CG-FAST coincided with all tested 
TABLE 2 | Comparison of baseline variables between LVOS and non-LVOS patients.

\begin{tabular}{|c|c|c|c|}
\hline & $\begin{array}{c}\text { LVOS } \\
(n=664)\end{array}$ & $\begin{array}{c}\text { Non-LVOS } \\
(n=691)\end{array}$ & $P$-value \\
\hline Male $(n, \%)$ & $380(57.2 \%)$ & $441(63.8 \%)$ & 0.013 \\
\hline Age (year) & $70 \pm 12$ & $68 \pm 13$ & 0.001 \\
\hline Prior antiplatelet therapy $(n, \%)$ & $124(18.7 \%)$ & $121(17.5 \%)$ & 0.578 \\
\hline Smoking $(n, \%)$ & $194(29.2 \%)$ & $234(33.9 \%)$ & 0.066 \\
\hline Hypertension (n, \%) & $456(68.7 \%)$ & $464(67.1 \%)$ & 0.548 \\
\hline Diabetes mellitus ( $n, \%)$ & $129(19.4 \%)$ & $171(24.7 \%)$ & 0.018 \\
\hline Hyperlipidemia (n, \%) & 241 (36.3\%) & $284(41.1 \%)$ & 0.070 \\
\hline History of stroke/TIA (n, \%) & 119 (17.9\%) & 135 (19.5\%) & 0.446 \\
\hline Atrial fibrillation (n, \%) & 317 (47.7\%) & $157(22.7 \%)$ & $<0.001$ \\
\hline Systolic blood pressure (mmHg) & $153 \pm 27$ & $159 \pm 25$ & $<0.001$ \\
\hline Glucose (mmol/L) & $7.6 \pm 2.6$ & $7.9 \pm 2.9$ & 0.025 \\
\hline NIHSS sum & $13(8-18)$ & $4(2-10)$ & $<0.001$ \\
\hline \multicolumn{4}{|l|}{ LOC } \\
\hline A) LOC responsiveness & $0(0-1)$ & $0(0-0)$ & $<0.001$ \\
\hline B) LOC questions & $1(0-2)$ & $0(0-0)$ & $<0.001$ \\
\hline C) LOC commands & $0(0-2)$ & $0(0-0)$ & $<0.001$ \\
\hline Gaze deviation & $1(0-2)$ & $0(0-0)$ & $<0.001$ \\
\hline Visual field test & $0(0-0)$ & $0(0-0)$ & 0.800 \\
\hline Facial palsy & $1(1-2)$ & $1(0-1)$ & $<0.001$ \\
\hline Motor arm & $4(2-4)$ & $0(0-3)$ & $<0.001$ \\
\hline Motor left arm & $0(0-4)$ & $0(0-1)$ & $<0.001$ \\
\hline Motor right arm & $0(0-4)$ & $0(0-1)$ & $<0.001$ \\
\hline Motor leg & $3(2-4)$ & $1(0-3)$ & $<0.001$ \\
\hline Motor left leg & $0(0-3)$ & $0(0-1)$ & $<0.001$ \\
\hline Motor right leg & $0(0-3)$ & $0(0-1)$ & $<0.001$ \\
\hline Limb ataxia & $0(0-0)$ & $0(0-0)$ & 0.002 \\
\hline Sensory & $0(0-1)$ & $0(0-1)$ & 0.123 \\
\hline Aphasia & $2(0-3)$ & $0(0-1)$ & $<0.001$ \\
\hline Dysarthria & $1(0-2)$ & $1(0-1)$ & $<0.001$ \\
\hline Extinction and inattention & $0(0-0)$ & $0(0-0)$ & 0.003 \\
\hline \multicolumn{4}{|l|}{ INFARCTION LOCALIZATION } \\
\hline Anterior circulation & $594(89.5 \%)$ & $591(85.5 \%)$ & 0.029 \\
\hline Posterior circulation & $61(9.2 \%)$ & $81(11.7 \%)$ & 0.128 \\
\hline Both involved & $9(1.4 \%)$ & $19(2.7 \%)$ & 0.071 \\
\hline \multicolumn{4}{|l|}{ OCCLUSION SITES } \\
\hline ICA & $196(29.5 \%)$ & - & - \\
\hline M1 & 452 (68.1\%) & - & - \\
\hline M2 & $32(4.8 \%)$ & - & - \\
\hline BA & 62 (9.3\%) & - & - \\
\hline
\end{tabular}

LOC, indicates level of consciousness; LVOS, large vessel occlusion strokes; NIHSS, national institutes of health stroke scale; ICA, intracranial internal carotid artery; M1, M1 segment of the middle cerebral artery; M2, M2 segment of the middle cerebral artery; and $B A$, basilar artery.

items as an acronym, which could be easily memorized by emergency paramedics.

Ideally, it is better to use different pre-hospital triage tools with different predictive values for LVOS according to the location of CSC. If a CSC is very close to the patient, then a triage tool with

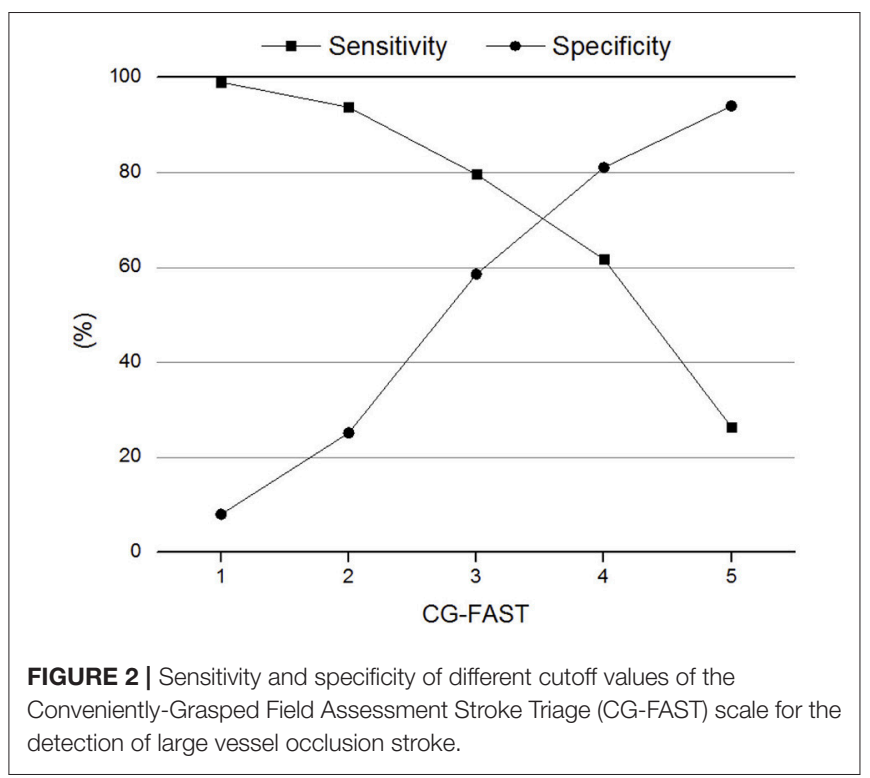

high sensitivity is more appropriate because we need to avoid missing the possible LVOS patients. For distal CSC, a triage tool with high specificity is better so that patients with low possibility of LVOS do not need to waste time on the road. However, this will obviously increase the operational complexity for emergency paramedics, so it is seldom used in real world practice.

Our study has some limitations. First, the scale was derived and validated from a single cohort. The retrospective and observational design inherits potential for bias. Our results need to be confirmed prospectively in larger cohorts. Second, all patients were diagnosed in our center. The predictive value of CG-FAST for LVOS may be different in the pre-hospital settings because it will inevitably mix with stroke mimics or hemorrhagic strokes. Thus, studies performed in a preclinical setting are necessary to generalize our results. Third, the elements derived from the NIHSS were completed at the time of admission to the hospital by experienced neurologists rather than emergency paramedics in the field, although it was proven that NIHSS had good inter-observer reproducibility between physicians and other health personnel $(31,32)$ and we have already designed our scale to be easily assessed by emergency paramedics. Finally, prehospital triage tools could assist emergency paramedics to make rough judgments and transfer appropriate patients to a CSC in a timely manner. But we should realize that no scale could take the place of vessel imaging $(33,34)$. Therefore, MRA or CTA is better to be performed in all patients suspected of LVOS as soon as possible.

In summary, the CG-FAST scale could be an effective and simple tool to identify patients with high likelihood of LVOS, which might help to select eligible patients for EVT and transfer appropriate patients to a CSC quickly. The utility and accuracy of the CG-FAST scale to predict LVOS in the pre-hospital setting needs to be tested in further prospective studies. 
TABLE 3 | Comparison of thresholds of the pre-hospital stroke scales, CG-FAST, and NIHSS according to AUC, sensitivity, specificity, PPV and NPV, and accuracy.

\begin{tabular}{|c|c|c|c|c|c|c|c|c|c|}
\hline Scale & AUC & Youden index & SEN & SPE & PPV & NPV & Accuracy & $95 \% \mathrm{Cl}$ & $P$-value \\
\hline FAST-ED $\geq 3$ & 0.747 & 0.401 & 0.735 & 0.666 & 0.714 & 0.737 & 0.725 & $0.719-0.776$ & $<0.001$ \\
\hline $3-I S S \geq 3$ & 0.729 & 0.388 & 0.587 & 0.801 & 0.771 & 0.680 & 0.714 & $0.701-0.758$ & $<0.001$ \\
\hline $\mathrm{CPSSS} \geq 2$ & 0.751 & 0.414 & 0.693 & 0.721 & 0.740 & 0.722 & 0.730 & $0.723-0.779$ & $<0.001$ \\
\hline$P A S S \geq 2$ & 0.739 & 0.417 & 0.696 & 0.721 & 0.739 & 0.723 & 0.731 & $0.711-0.768$ & $<0.001$ \\
\hline RACE $\geq 5$ & 0.740 & 0.407 & 0.675 & 0.732 & 0.737 & 0.709 & 0.722 & $0.711-0.768$ & $<0.001$ \\
\hline$L A M S \geq 3$ & 0.709 & 0.345 & 0.785 & 0.560 & 0.663 & 0.696 & 0.528 & $0.680-0.739$ & $<0.001$ \\
\hline G-FAST $\geq 3$ & 0.715 & 0.315 & 0.751 & 0.564 & 0.660 & 0.721 & 0.687 & $0.685-0.744$ & $<0.001$ \\
\hline CG-FAST $\geq 4$ & 0.758 & 0.428 & 0.617 & 0.810 & 0.785 & 0.692 & 0.728 & $0.731-0.786$ & $<0.001$ \\
\hline $\mathrm{NIHSS} \geq 9$ & 0.746 & 0.416 & 0.732 & 0.684 & 0.719 & 0.264 & 0.490 & $0.718-0.775$ & $<0.001$ \\
\hline $\mathrm{NIHSS} \geq 6^{*}$ & 0.746 & 0.307 & 0.818 & 0.489 & 0.641 & 0.761 & 0.686 & $0.718-0.775$ & $<0.001$ \\
\hline
\end{tabular}

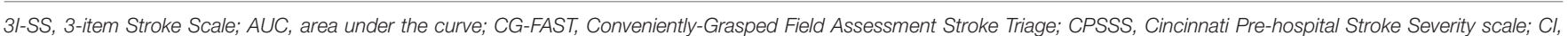

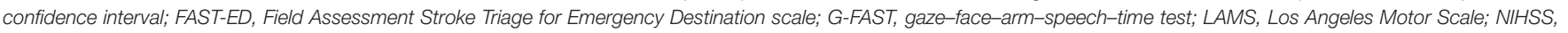

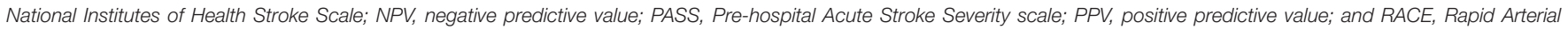
Occlusion Evaluation Scale; SEN, Sensitivity; SPE, Specificity.

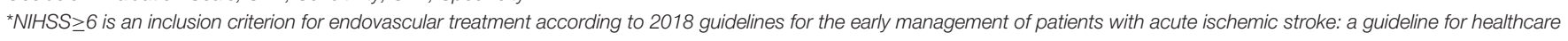
professionals from the American Heart Association/American Stroke Association (16).

\section{ETHICS STATEMENT}

All subjects had given written informed consent prior to the study, and the protocols had been approved by the human ethics committee of the second affiliated hospital of Zhejiang University, School of Medicine. Clinical investigation had been conducted according to the principles expressed in the Declaration of Helsinki.

\section{AUTHOR CONTRIBUTIONS}

XG and ZC drafted and revised the manuscript, participated in study concept and design, conducted the statistical analyses,

\section{REFERENCES}

1. Berkhemer OA, Fransen PS, Beumer D, van den Berg LA, Lingsma HF, Yoo AJ, et al. A randomized trial of intraarterial treatment for acute ischemic stroke. N Engl J Med. (2015) 372:11-20. doi: 10.1056/NEJMoa1411587

2. Goyal M, Demchuk AM, Menon BK, Eesa M, Rempel JL, Thornton J, et al. Randomized assessment of rapid endovascular treatment of ischemic stroke. N Engl J Med. (2015) 372:1019-30. doi: 10.1056/NEJMoa1414905

3. Campbell BC, Mitchell PJ, Kleinig TJ, Dewey HM, Churilov L, Yassi N, et al. Endovascular therapy for ischemic stroke with perfusion-imaging selection. $N$ Engl J Med. (2015) 372:1009-18. doi: 10.1056/NEJMoa1414792

4. Saver JL, Goyal M, Bonafe A, Diener HC, Levy EI, Pereira VM, et al. Stentretriever thrombectomy after intravenous t-PA vs. t-PA alone in stroke. $\mathrm{N} \mathrm{Engl}$ J Med. (2015) 372:2285-95. doi: 10.1056/NEJMoa1415061

5. Jovin TG, Chamorro A, Cobo E, de Miquel MA, Molina CA, Rovira A, et al. Thrombectomy within 8 hours after symptom onset in ischemic stroke. N Engl J Med. (2015) 372:2296-306. doi: 10.1056/NEJMoa1503780

6. Nogueira RG, Jadhav AP, Haussen DC, Bonafe A, Budzik RF, Bhuva P, et al. Thrombectomy 6 to 24 hours after stroke with a mismatch between deficit and infarct. N Engl J Med. (2018) 378:11-21. doi: 10.1056/NEJMoa17 06442

7. Lemmens R, Hamilton SA, Liebeskind DS, Tomsick TA, Demchuk AM, Nogueira RG, et al. Effect of endovascular reperfusion in analyzed and interpreted the data. ML participated in study concept and design, data interpretation and made a major contribution in revising the manuscript. FS, MZ, CX, and RZ participated in the study design and made contribution in revising the manuscript.

\section{FUNDING}

This study was supported by the National Natural Science Foundation of China (81622017, 81471170 and 81601017), the National Key Research and Development Program of China (2016YFC1301503), the Science Technology Department of Zhejiang Province (2018C04011) and Fundamental Research Funds for the Central Universities (2017XZZX002-09).

relation to site of arterial occlusion. Neurology. (2016) 86:762-70. doi: 10.1212/WNL.0000000000002399

8. Sarraj A, Sangha N, Hussain MS, Wisco D, Vora N, Elijovich L, et al. Endovascular therapy for acute ischemic stroke with occlusion of the middle cerebral artery M2 segment. JAMA Neurol. (2016) 73:1291-6. doi: 10.1001/jamaneurol.2016.2773

9. Gory B, Eldesouky I, Sivan-Hoffmann R, Rabilloud M, Ong E, Riva R, et al. Outcomes of stent retriever thrombectomy in basilar artery occlusion: an observational study and systematic review. J Neurol Neurosurg Psychiatry. (2016) 87:520-5. doi: 10.1136/jnnp-2014-310250

10. Prabhakaran S, Ruff I, Bernstein RA. Acute stroke intervention: a systematic review. JAMA. (2015) 313:1451-62. doi: 10.1001/jama.2015.3058

11. Emberson J, Lees KR, Lyden P, Blackwell L, Albers G, Bluhmki E, et al. Effect of treatment delay, age, and stroke severity on the effects of intravenous thrombolysis with alteplase for acute ischaemic stroke: a meta-analysis of individual patient data from randomised trials. Lancet. (2014) 384:1929-35. doi: 10.1016/S0140-6736(14)60584-5

12. Ismail M, Armoiry X, Tau N, Zhu F, Sadeh-Gonik U, Piotin M, et al. Mothership versus drip and ship for thrombectomy in patients who had an acute stroke: a systematic review and meta-analysis. J Neurointerv Surg. (2019) 11:14-9. doi: 10.1136/neurintsurg-2018-014249

13. Smith EE, Saver JL, Cox M, Liang L, Matsouaka R, Xian Y, et al. Increase in endovascular therapy in get with the guidelines-stroke 
after the publication of pivotal trials. Circulation. (2017) 136:2303-10. doi: 10.1161/CIRCULATIONAHA.117.031097

14. Lima FO, Silva GS, Furie KL, Frankel MR, Lev MH, Camargo EC, et al. Field assessment stroke triage for emergency destination: a simple and accurate prehospital scale to detect large vessel occlusion strokes. Stroke. (2016) 47:1997-2002. doi: 10.1161/STROKEAHA.116. 013301

15. Noorian AR, Sanossian N, Shkirkova K, Liebeskind DS, Eckstein M, Stratton SJ, et al. Los Angeles motor scale to identify large vessel occlusion: prehospital validation and comparison with other screens. Stroke. (2018) 49:565-72. doi: 10.1161/STROKEAHA.117.019228

16. Powers WJ, Rabinstein AA, Ackerson T, Adeoye OM, Bambakidis NC, Becker K, et al. 2018 Guidelines for the early management of patients with acute ischemic stroke: a guideline for healthcare professionals from the american heart association/american stroke association. Stroke. (2018) 49:e46-110. doi: $10.1161 /$ STR.0000000000000158

17. Vanacker P, Heldner MR, Amiguet M, Faouzi M, Cras P, Ntaios G, et al. Prediction of large vessel occlusions in acute stroke: national institute of health stroke scale is hard to beat. Crit Care Med. (2016) 44:e336-43. doi: 10.1097/CCM.0000000000001630

18. Cooray C, Fekete K, Mikulik R, Lees KR, Wahlgren N, Ahmed N. Threshold for NIH stroke scale in predicting vessel occlusion and functional outcome after stroke thrombolysis. Int J Stroke. (2015) 10:822-9. doi: 10.1111/ijs. 12451

19. Heldner MR, Zubler C, Mattle HP, Schroth G, Weck A, Mono ML, et al. National Institutes of Health stroke scale score and vessel occlusion in 2152 patients with acute ischemic stroke. Stroke. (2013) 44:1153-7. doi: 10.1161/STROKEAHA.111.000604

20. Katz BS, McMullan JT, Sucharew H, Adeoye O, Broderick JP. Design and validation of a prehospital scale to predict stroke severity: cincinnati prehospital stroke severity scale. Stroke. (2015) 46:1508-12. doi: 10.1161/STROKEAHA.115.008804

21. Singer OC, Dvorak F, du Mesnil de Rochemont R, Lanfermann H, Sitzer M, Neumann-Haefelin T. A simple 3-item stroke scale: comparison with the National Institutes of Health Stroke Scale and prediction of middle cerebral artery occlusion. Stroke. (2005) 36:773-6. doi: 10.1161/01.STR.0000157591.61322.df

22. Nazliel B, Starkman S, Liebeskind DS, Ovbiagele B, Kim D, Sanossian N, et al. A brief prehospital stroke severity scale identifies ischemic stroke patients harboring persisting large arterial occlusions. Stroke. (2008) 39:2264-7. doi: 10.1161/STROKEAHA.107. 508127

23. Perez de la Ossa N, Carrera D, Gorchs M, Querol M, Millan M, Gomis M, et al. Design and validation of a prehospital stroke scale to predict large arterial occlusion: the rapid arterial occlusion evaluation scale. Stroke. (2014) 45:87-91. doi: 10.1161/STROKEAHA.113.003071

24. Hastrup S, Damgaard D, Johnsen SP, Andersen G. Pre-hospital acute stroke severity scale to predict large artery occlusion: design and comparison with other scales. Stroke. (2016) 47:1772-6. doi: 10.1161/STROKEAHA.115. 012482

25. Scheitz JF, Abdul-Rahim AH, MacIsaac RL, Cooray C, Sucharew $\mathrm{H}$, Kleindorfer $\mathrm{D}$, et al. Clinical selection strategies to identify ischemic stroke patients with large anterior vessel occlusion: results from SITS-ISTR (Safe Implementation of Thrombolysis in Stroke International Stroke Thrombolysis Registry). Stroke. (2017) 48:290-7. doi: 10.1161/STROKEAHA.116.014431

26. Chen Z, Zhang R, Xu F, Gong X, Shi F, Zhang M, et al. Novel prehospital prediction model of large vessel occlusion using artificial neural network. Front Aging Neurosci. (2018) 10:181. doi: 10.3389/fnagi.2018.00181

27. Youden WJ. Index for rating diagnostic tests. Cancer. (1950) 3:32-5.

28. Tijssen CC, Schulte BP, Leyten AC. Prognostic significance of conjugate eye deviation in stroke patients. Stroke. (1991) 22:200-2.

29. Ringman JM, Saver JL, Woolson RF, Adams HP. Hemispheric asymmetry of gaze deviation and relationship to neglect in acute stroke. Neurology. (2005) 65:1661-2. doi: 10.1212/01.wnl.0000184494.88608.d1

30. Singer OC, Humpich MC, Laufs $H$, Lanfermann $H$, Steinmetz $H$, Neumann-Haefelin T. Conjugate eye deviation in acute stroke: incidence, hemispheric asymmetry, and lesion pattern. Stroke. (2006) 37:2726-32. doi: 10.1161/01.STR.0000244809.67376.10

31. Goldstein LB, Samsa GP. Reliability of the National Institutes of Health Stroke Scale. Extension to non-neurologists in the context of a clinical trial. Stroke. (1997) $28: 307-10$

32. Dewey HM, Donnan GA, Freeman EJ, Sharples CM, Macdonell RA, McNeil JJ, et al. Interrater reliability of the National Institutes of Health Stroke Scale: rating by neurologists and nurses in a community-based stroke incidence study. Cerebrovasc Dis. (1999) 9:323-7. doi: 10.1159/000016006

33. Turc G, Maier B, Naggara O, Seners P, Isabel C, Tisserand M, et al. Clinical scales do not reliably identify acute ischemic stroke patients with largeartery occlusion. Stroke. (2016) 47:1466-72. doi: 10.1161/STROKEAHA.116.0 13144

34. Heldner MR, Hsieh K, Broeg-Morvay A, Mordasini P, Buhlmann M, Jung S, et al. Clinical prediction of large vessel occlusion in anterior circulation stroke: mission impossible? J Neurol. (2016) 263:1633-40. doi: $10.1007 / s 00415-016-8180-6$

Conflict of Interest Statement: The authors declare that the research was conducted in the absence of any commercial or financial relationships that could be construed as a potential conflict of interest.

Copyright $\odot 2019$ Gong, Chen, Shi, Zhang, Xu, Zhang and Lou. This is an openaccess article distributed under the terms of the Creative Commons Attribution License (CC BY). The use, distribution or reproduction in other forums is permitted, provided the original author(s) and the copyright owner(s) are credited and that the original publication in this journal is cited, in accordance with accepted academic practice. No use, distribution or reproduction is permitted which does not comply with these terms. 